\title{
Data is the New Plastics: Developing Machine Learning UX Design Methods for Artificial Intelligence
}

\author{
Frédérique Krupa \\ l'école de design Nantes Atlantique \\ *f.krupa@lecolededesign.com
}

\begin{abstract}
:
Designers' next technological frontier is the creation of artificial intelligence (Al) for and within design systems. Al deployed in products, services and systems make use of Adrian Forty's (1986) Suppressive design strategy to reduce consumer resistance to technological progress. Al production is currently the exclusive domain of data scientists and engineers, and a narrow form of $\mathrm{Al}$ is produced through machine learning (ML) algorithms that extract patterns from data in order to automate cognitive processes like predictions, categorizations, clustering, pathfinding, optimizations and more. ML's homogenous production teams urgently needs diversifying, but machine learning's algebraic and statistical foundations serve as intimidating gatekeepers to this complex universe. However, ML is simply the new frontier that designers must engage in - like other technical domains that preceded it. Designing ML-enhanced user experiences requires the development of new design pedagogy and research methods, hybridizing design with engineering and social sciences. The designer's role on a production team, as well as the granularity and focus of a designer's technical understanding, are currently up for debate. We proposes an approach called MLUX (Machine Learning User Experience), a syllabus for designing user experiences (UX) for ethical, responsible Al-based systems.
\end{abstract}

Keywords: MLUX, machine Learning user experience, artificial intelligence design, data science, suppressive design

\section{The New Oil or the New Plastics?}

While some believe that "data is the new oil" (Economist 2017), plastics is a more appropriate metaphor. Like plastics, data is the result of significant human intervention, not a naturally occurring resource, and its true value is derived from its adaptation to human needs. Like the plastics found in the Great Pacific Garbage Patch, the gross accumulation of unstructured big data, reported to be sitting unused on webserver farms, poses a serious ecological threat. Like plastics, data can be used 
in ways that are potentially beneficial (e.g. convenient, protective, etc.) and harmful (e.g. toxic, invasive, etc.). Like the introduction of plastics to the designers' material repertoire in the mid $20^{\text {th }}$ century, data is the new immaterial material that designers need to apprehend.

\subsection{Design Magic, Materials \& Processes}

Design, like technology, is a group endeavor requiring the organization and collaboration of technical specialists. Design, like technology, is a social construct whose goals, values and ideals are defined through its social context or milieu. In a capitalist system, design is critical to overcoming fear and resistance to technological innovation (Forty 1986). Design's ultimate success has been its ability to control user experience, not unlike a magician that guides an audience's attention away from the real action. Design's "sleight of hand" has always been in the service of capitalism, focusing attention and emotional response (Norman 2004) when and where necessary to not only delight and serve, but to overcome consumer resistance to novelty and enable progress.

In Objects of Desire, Adrian Forty (1986, p 11-13) identifies three technological commodification strategies - Archaic, Suppressive and Utopian design. Throughout the industrial revolution and up to early modernism, the Archaic approach prevailed, using formal design references of the past as an "antidote to progress," to reduce consumer anxiety and resistance to technology at a time of great social instability. In the $20^{\text {th }}$ Century, consumer fears gave way to a more celebratory acknowledgement of technology's benefits, and the Utopian approach commodified technology using formal design references to a future and better world. Suppressive design, the third and least frequent strategy, denies the presence of technology by making it invisible or embedding it in a generic object like a speaker, smartphone, watch, doorbell or television. Suppressive design has become the principal strategy for designing Al-enhanced user experiences by embedding machine learning prediction capabilities into "smart" common objects or behind the scenes of our digital interactions. Suppressive design masks or hides technologically-advanced functions to decrease consumer resistance at a time when privacy and automated decision-making are coming under increasing scrutiny.

Unbridled technological solutionism and hype-filled Al marketing have been met with increasing pushback, not only from the public (Chuisi \& al. 2020) but from the scientific community as well (Middlestadt 2019). Bans on the use of facial recognition technology in certain cities or for police departments, Europe's GDPR, the Cambridge Analytica scandal, and well publicized examples of racist and sexist algorithms (Wachter-Boettcher 2017, Costanza-Chock 2020) have created an apprehension towards the abuse and misuse of personal data and automated decision-making. This consumer skepticism will only increase until proper and enforceable regulatory frameworks are developed, but as recent debates on ethical frameworks have shown (IEEE 2019, Jobin \& al. 2019, Middlestadt 2019), they are still a long way off.

Therefore, when Al functions according to plan, it is often embedded within a system so as to make its influence imperceptible or forgotten, such as when we navigate with GPS-enabled smartphones, read spam-free emails, browse social media, shop online, or watch a video-on-demand service. If interactions are necessary, Al often masks its presence through affective computing strategies (Picard 2020) that provides anthropomorphic traits like voice user interfaces (VUI) in the digital assistants Siri (Apple), Cortana (Microsoft) and Alexa (Amazon). Disguising, concealing and transforming $\mathrm{Al}$ in relatively banal connected objects conveniently makes us forget the risks that they may bring into our lives:

“... by codifying and entrenching biases, reducing accountability, hindering due process, and increasing the information asymmetry between individuals whose 
data feed into these systems and big players capable of inferring potentially relevant information." (ACM FAccT 2020)

Since machine learning models are increasingly supplying the "intelligence" in complex systems, the tendency is to keep their process and results as "natural" and unobtrusive as possible, creating a Suppressive system design.

\subsection{Machine Learning Design}

As design has grown in systemic complexity - from traditional materials and processes of industrial production to digital technology - from products to services to hybrid systems, so have the designers adjusted their design methodologies and incorporated new research methods. To deal with systemic complexity, these new design research methods often borrowed from tangential "soft" science fields like sociology, psychology, anthropology, communications or marketing, along with the "hard" science of engineering. Design's appropriation of qualitative social science methods is a major asset that designers bring to research and development teams, along with stake-holder-centric design methods. (Krupa \& al. 2020) UX design in particular, spun off from user interaction design (UI) to contend with user experiences in a more holistic manner, identifying the conflicts of interest that may arise from an increasingly complex network of stakeholders.

The complexity of machine learning technology and its dematerialized presence and influence create an important digital divide between designers and the data scientists and engineers that currently construct these systems. Engineering-led ML production teams are homogenous in terms of gender, ethnicity, social class, etc. The people making ML technology come from the most privileged segments of society and are the least likely to suffer negative consequences of biased technology. Diversifying production teams is an imperative, but this has proven quite difficult to achieve (Krupa 2018). Machine learning remains engineering-driven, focused almost exclusively on algorithms, relegating concerns about data quality, bias and user experience to the back burner. In other words, the industry is relatively immature, still driven by the ethos "move fast, break things and ask questions later." Bringing in UX designers is one way to diversify ML production teams, by encouraging interdisciplinary diversity.

\section{Machine Learning User Experience Design}

Let us return to the definitions and differences between Al and ML as explained by Chollet (2020), Google engineer and creator of the Keras neural networks library. Artificial general intelligence (AGI) depicted in movies like Terminator requires autonomous abstraction generation and therefore exists only in our imaginations. What exists is a narrow $\mathrm{Al}$, a form of cognitive automation, while $\mathrm{ML}$ is the encoding of implicit abstractions. He explains in greater detail:

" Our field isn't quite "artificial intelligence" -- it's "cognitive automation": the encoding and operationalization of human-generated abstractions/behaviors/skills. The "intelligence" label is a category error...

Intelligence is adaption to unknown unknowns across an unknown range of tasks and domains. Automation is, at best, robustly handling known unknowns over known tasks (which is already incredibly difficult and resource-intensive in the real world -- whether engineering or data).» (Chollet 2020)

While designers have engaged in industrial production since the division of labor (Forty 1986), the engineers and data scientist on ML development teams are beginning to acknowledge that the questions being posed by civil society regarding Al bias and harms fall beyond the scope of their 
training, which is focused on advancing technology. While it might seem less of a stretch to instill user-centered design methods for data scientists than instilling machine learning understanding for designers, the relative youth and rapid development of machine learning technology burdens data scientists and engineers with the overwhelming injunction to focus on feasibility, performance and optimization. UX designers are trained to look for and understand stakeholder's conflicts of interest in a system, and this makes them particularly apt to join a creative team and development process (Krupa \& al. 2020). In ML development, UX design methods like critical design, design fiction, or userneed hierarchies can be used to identify and predict adverse effects on minority "outliers" and the potential bad actors. The opportunity lies for designers to join data scientists and engineers as long as they can harness the necessary concepts and skills to be competent ML production team members; however, these tools and methods have trouble rising up in UX designers' best practices (Yang 2018c). In particular, consensus is lacking regarding:

- What ML skills should be developed in designers,

- What ML branches should designers focus on and to what level of detail, and

- What roles and interventions does the designer take on relative to the data scientist, and how does this impact their education?

These are the issues that currently underpin the slow development of an MLUX curriculum. This paper discusses two iterations of a syllabus trying to address these issues that took place in a UX design master's program.

\subsection{Granularity, focus, and roles}

$\mathrm{ML}$ is rapidly evolving field whose advances are not discrete algorithmic approaches. Instead, optimal results often come from sequential and hybridized algorithmic approaches. Figure 1 explains the main branches of machine learning and their principal applications, which begs the following questions: Which branches should be focused upon, and what level of technical granularity must be achieved for deep conceptual understanding and mastery? 


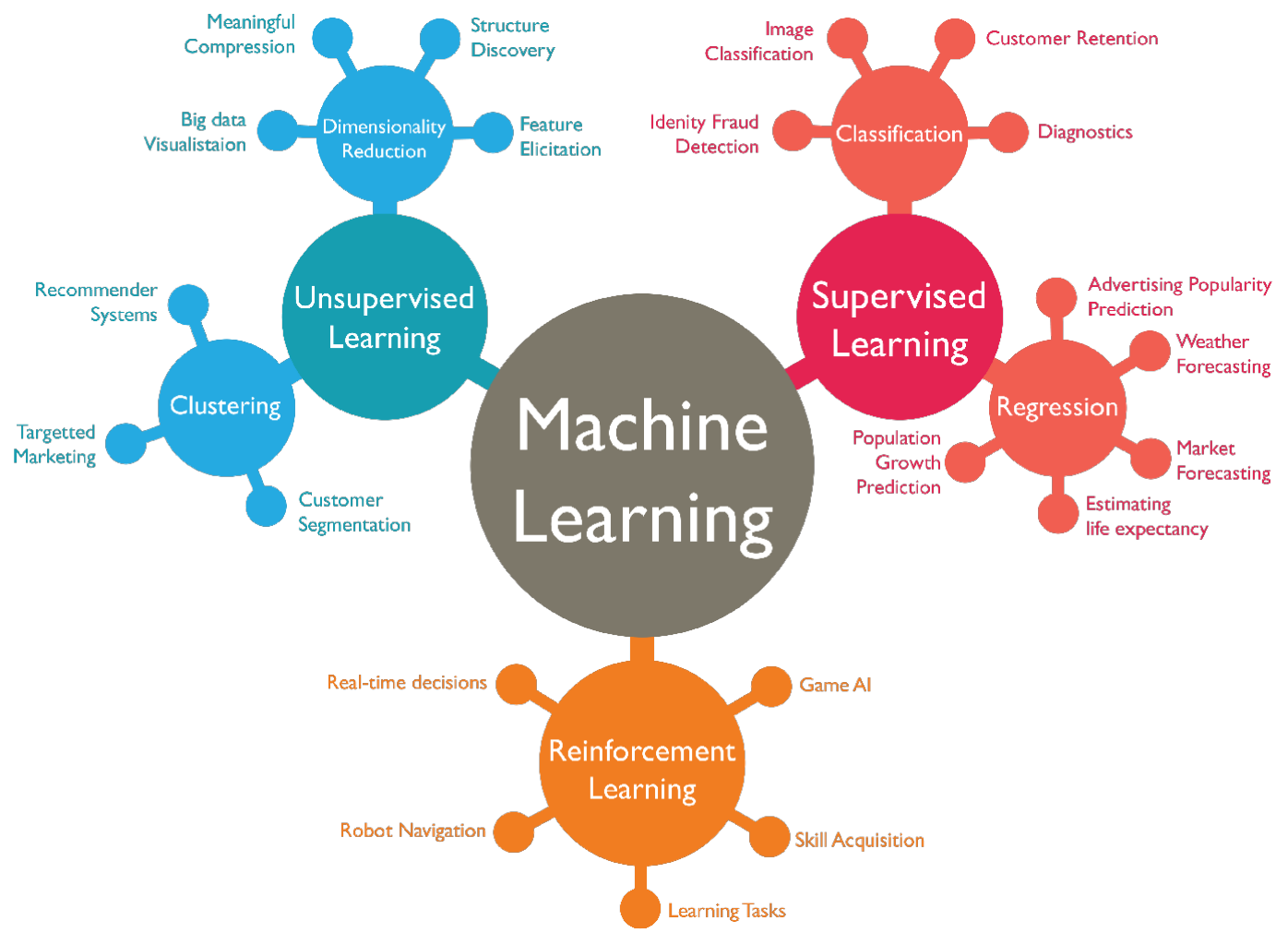

Figure 1. Main Branches of Machine Learning. Note: Neural networks (deep learning) is a subset of machine learning. Source: askdatascience.com/13/what-are-the-main-branches-of-machine-learning (https://i.imgur.com/rY1jjwx.png)

Computer science programs like 42Al often start by focusing on regression algorithms used for forecasting and predictions because they are the easiest to explain (Krupa 2020). For the past few years, MLUX has developed as a course at the Stanford d.School (Carney 2018, 2019), Carnegie Mellon (Yang \& al. 2018b), and Loughborough University. Schools often focus on theory and issues of bias, accountability and harms. If prototyping is attempted, the complex algorithmic pipeline is delegated to more user-friendly commercial packages like Microsoft Azure, IBM Watson, or Amazon Web Services. While this black box approach to ML is accessible for many designers, it relinquishes deep technical understanding and control to data scientists and keeps designers at the periphery. Yang \& al. (2018a, 2018b) and Dove \& al. (2017) identified that this is how experienced UX designers currently work with machine learning, but designer's lack of technical mastery is keeping ML development technology-led rather than design-led.

Without understanding how data patterns are being mathematically extracted, evaluated, and simplified, designers are in the audience of a magic show. Quantifying the messy complexity of data is not a neutral act, and this flattening of reality is well described in the $\mathrm{CHI} 2021$ paper presentation of Alkhatib (2021), director of the Center of Applied Data Ethics:

"The rules machine learning systems infer from the data have no underlying meaning or reason behind them. They're just patterns, without any insight into *why* Black people are in prison at much higher rates than white people (for instance). There's no dataset in the world that adequately conveys white supremacy, slavery, and colonialism. So at best these systems generate a facsimile of a world with the shadows of history cast on the ground - skewed, flattened, and always lacking depth that only living these experiences can bring. These rules are devoid of meaning, but in the world the Al has constructed, everything makes a kind of sense. And they punish or reward us for fitting into the model they generate - in other words, the world they construct." (Alkhatib 2021) 
Or as Langdon Winner would say, "artifacts have politics." (Winner 1980).

\subsection{MLUX Design V1}

The case-study presented here is of the development of an MLUX syllabus that provides an optional technical course within a two year UX Design Master's program at the école de design Nantes Atlantique in France. Our program comprises the fourth and fifth year in a five year design program. The Master's program focuses on interdisciplinary group-based industry-sponsored projects with a strong UX and user interaction (UI) component. Our students come from different undergraduate disciplines like interaction design, product design, transportation, motion graphics or scenography. Our department tends to attract designers with a strong interest in technology and/or with advanced technical capabilities like programming and prototyping skills. While the core of the program focuses on a common UX design curriculum, students may specialize through optional technical workshop. MLUX is the newest technical specializations, proposed alongside workshops in tangible and immersive user-experience technologies.

For our initial deep dive into the algorithmic process, we adapted 42Al's 14 week syllabus using its peer-to-peer pedagogy (Krupa 2020) to learn key ML concepts, methods, applications, workflows and approaches - stressing pragmatic and conceptual understanding of feature extraction and developing intuition for the development process through a reverse engineering approach. This syllabus was structured in a two semester plan as follows:

\section{Semester 1}

- Intro to $\mathrm{ML}+$ Python

- Simple Linear Regression + Linear Algebra + Numpy

- Multiple Linear Regression + Derivatives/Gradient + Pandas

- Logistic Regression

- Support Vector Machines

- Decision Trees + Random Forest + Boosted Tree

- Naive Bayes

- Unsupervised (PCA \& K-Means)

- Recommender Systems

- Neural Networks (Intro)

- Convolutional Neural Networks (CNN)

- Recurrent Neural Networks (RNN)

- Reinforcement Learning + Q-learning

- Deep Reinforcement learning (DQN + Policy Gradients)

The subsequent semester would focus on understanding ML's theoretical and ethical underpinnings and its link to UX design practice, through scientific literature, creative coding exercises and reflexive thinking.

\section{Semester 2}

- Data Collection and Data Science Workflows

- Data Visualization and Exploration

- Recommender Systems and UX

- Natural Language Processing and Voice Interfaces (Chatbots and VUI)

- Computer Vision (Neural Networks and High Dimensional Classifiers)

- Image \& Video Processing, Transfer Learning 
- Generative Design \& GANs

- Disruption, Innovation, Ethics and Bias

\section{Results}

In 2019-2020, the Covid-19 pandemic and subsequent school closures forced us to significantly reduce our ambitions to 6 reverse-engineering sessions with a remote instructor. 18 students began the series of technical workshops, 6 students followed the workshop assiduously, and only two completed all six workshops. The hands-on workshop used the Python programming language in Jupyter Notebook. The first students to abandon the workshop were those with little to no programming experience. Some students that had not had a strong mathematical foundation in high school abandoned due to the time constraint of catching up on linear algebra, statistics, and matrix calculations. One mitigating factor was that this workshop came in addition to regular coursework, and some abandoned to focus on their thesis projects instead.

Since normal educational conditions were not re-established, subsequent technical sessions were postponed. After only the six sessions, the student attrition rate incited us to restructure the approach to mix theory and technical components more directly.

\subsection{MLUX Design V2}

In 2020-2021, the MLUX V2 syllabus was adapted into a shorter, siloed approach - attempting to link theory about ML ethics, innovation and bias directly to hands-on practice in a specific algorithmic approach. This linking of technical understanding to design considerations was more successful with students, and more accommodating to the pandemic's scheduling turmoil and degraded pedagogical context because, aside from the introductory workshop, the sessions could be scheduled and attended out of sequence. In the end, only the first three out of eight workshop topics were successfully scheduled in this year, with the remaining five to be completed next year.

Below is the proposed MLUX V2 structure, along with the instructor contact hours in parentheses, whereby four contact hours with students is scheduled per day.

- Introduction to ML algorithmic design + Python, Jupyter Notebook, Numpy, Pandas (16 hours)

Simple linear regression + Linear algebra

Multiple Linear Regression + Derivatives/Gradient Descent Logistic regression

- Data collection and workflow in data science - (8 hours) Data visualization \& exploration (optional: dimensionality reduction)

- Natural Language Processing \& speech interfaces - (16 hours) (transformers, sentiment analysis, spam/hate speech detection, etc.) Speak UX: chatbot UX design workshop

- Computer vision (neural networks \& high-dimensional classifiers) - (16 hours) Facial recognition, emotion detection

- Recommender systems \& UX (clustering) - (4 hours)

- Image and video processing, transfer learning - (16 hours) Generative design, GANs (deepfakes)

- Reinforcement learning, wayfinding for robots and game Al- (8 hours) 


\section{Results}

Students appreciated the hands-on, project-based approach, and no attrition was observed on multiday sessions. However, reliance on black box ML prototyping solutions like Voiceflow or RunwayML reduced the student's depth of $M L$ technical understanding by keeping its algorithmic pipeline well hidden. The risks of bias associated with using pre-weighted models were also not evident through this approach. While design students remained engaged throughout the various workshops, the reduction of ML complexity keeps its potential for bias and harm hidden as well, defeating one of the principal goals of the workshop. Student engagement came at the price of reduced technical competence and ultimately, not being disruptive of the power-balance in an engineering-led production team.

\section{Conclusion}

The pandemic created difficult, artificial conditions for curricular development, making the evaluation of these two different pedagogical structures inconclusive. Since the students from MLUX V1 are only entering the job market now, it is too early to gauge the impact of these workshops on their careers choices or prospects; however, some interesting take-aways from these different approaches were noted. In the first iteration of MLUX, students that followed the sessions assiduously were students that had programming experience or strong UI prototyping skills, as well as solid math skills from high school (France's Bac S). Other student profiles were quickly discouraged by the technical and mathematical complexity, even if they had expressed a desire to learn how ML is produced. In the second version of MLUX, there was wider adoption of black box prototyping tools, who are improving in quality, but the students clearly did not understand the finer technical points like dataset feature extraction. This broad but shallow approach still leaves designers dependent on the technical team, and keeps ML firmly engineering-driven.

Hence, if we wish for a design-led ML, we still need to deepen the technical understanding of designers, which may be a path reserved for the most diligent programming-and-math-loving designers amongst us. Or perhaps, we are seeing the birth of a new profession, the UX designerengineer or the UX designer-data scientist, opening new questions about the interdisciplinarity between engineering, design and social sciences.

\section{References}

ACM FAccT (2020). ACM Conference on Fairness, Accountability, and Transparency. Retrieved April 30, 2021: https://facctconference.org/index.html

Alkhatib, A. (2021, May). To live in their utopia: Why algorithmic systems create absurd outcomes. In Proceedings of the 2021 CHI Conference on Human Factors in Computing Systems (pp. 1-9). Transcript of presentation retrieved 15/05/2021: https://t.co/zgM6E45nuz?amp=1

Carney, M. (2018) Designing machine learning. Stanford d.School (2018-2019). Course website consulted on 3/2/2020: https://designwith.ml/

Carney, M. (2019) Designing machine learning. Stanford d.School (2019-2020). Course website consulted on 3/2/2020: bit.ly/designingML2020

Chiusi, F. \& al. (Eds.) (2020). Automating Society Report 2020. Algorithm Watch, Bertelsmann Siftung. Retrieved April 30, 2021: https://automatingsociety.algorithmwatch.org

Chollet, F. (2020). https://twitter.com/fchollet/status/1214392496375025664 published on 07/01/2020. 
Costanza-Chock, S. (2020). Design justice: Community-led practices to build the worlds we need. The MIT Press.

Costanza-Chock, S. And Philip, N. (2018). Design justice, Al, and escape from the matrix of domination. Journal of Design and Science. 3(5). Retrieved 15/05/2021:

https://jods.mitpress.mit.edu/pub/costanza-chock/release/4

Dove, G., Halskov, K., Forlizzi, J., \& Zimmerman, J. (2017, May). UX design innovation: Challenges for working with machine learning as a design material. In Proceedings of the 2017 chi conference on human factors in computing systems (pp. 278-288).

Forty, A. (1986). Objects of desire: Design and society since 1750. Thames and Hudson.

IEEE Global Initiative on Ethics of Autonomous and Intelligent Systems. (2019). Ethically Aligned Design: A Vision for Prioritizing Human Well-being with Autonomous and Intelligent Systems, First Edition. Retrieved May 15, 2021: https://standards.iee.org/content/ieee-standards/en/industryconnections/ec/ autonomous-systems.html

Jobin, A., lenca, M., \& Vayena, E. (2019). The global landscape of Al ethics guidelines. Nature Machine Intelligence, 1(9), 389-399.

Krupa, F. (2018). Girl games: gender, design and technology in the service of women's recruitment in ICT?. PhD dissertation, the Université Paris 1 Panthéon-Sorbonne, 30/11/2018.

Krupa, F. (2020). The designer in the Al/machine learning creation process. Proceedings of CUMULUS ROMA 2021. June 9, 2021.

Krupa, F. \& al. (2020). L'UX Design: Livret des Méthodes. L'école de design Nantes Atlantique. https://www.lecolededesign.com/fichier/p_paralien/6610/livre_ux_digital_design_lab.pdf

Mittelstadt, B. (2019). Principles alone cannot guarantee ethical Al. Nature Machine Intelligence, 1(11), 501-507.

Norman, D. A. (2004). Emotional design: Why we love (or hate) everyday things. Basic Civitas Books.

Picard, R. W. (2000). Affective computing. MIT press.

The Economist (2017). The world's most valuable resource is no longer oil, but data. The Economist. May 6, 2017 online edition: https://www.economist.com/leaders/2017/05/06/the-worlds-mostvaluable-resource-is-no-longer-oil-but-data.

Wachter-Boettcher, S. (2017). Technically wrong: Sexist apps, biased algorithms, and other threats of toxic tech. WW Norton \& Company.

Winner, L. (1980). Do Artifacts have politics? Daedalus: Modern Technology: Problem or Opportunity?, 109(1), Winter 1980, 121-136.

Yang, Q., Scuito, A., Zimmerman, J., Forlizzi, J., \& Steinfeld, A. (2018a, June). Investigating how experienced UX designers effectively work with machine learning. In Proceedings of the 2018 Designing Interactive Systems Conference (pp. 585-596).

Yang, Q., Banovic, N., \& Zimmerman, J. (2018b, April). Mapping machine learning advances from hci research to reveal starting places for design innovation. In Proceedings of the $2018 \mathrm{CHI}$ Conference on Human Factors in Computing Systems (pp. 1-11).

Yang, Q. (2018c, March). Machine learning as a UX design material: How can we imagine beyond automation, recommenders, and reminders?. In AAAl Spring Symposia.

Author Bio:

Dr. Frédérique Krupa is the director of the Digital Design Lab at the école de design Nantes Atlantique. Her PhD dissertation Girl games: gender, design and technology in the service of women's recruitment in ICT? at the Université Paris 1 Panthéon-Sorbonne was followed by a postdoc action-research in peer-to-peer ICT pedagogy at the experimental computer science school 42 , where she specialized in machine learning algorithms and $\mathrm{Al}$ 
FREDERIQUE KRUPA

ethics. Her current research focuses on establishing MLUX methods, a holistic, transdisciplinary and systemic approach to create responsible machine learning data, algorithms and predictive models. 